\title{
Change in Mallampati Score during Pregnancy, Labour and Post Labour: An Observational Study
}

\author{
Archana Shivashankar ${ }^{*}$, Geetha C Rajappa ${ }^{2}$, Prapti Rath ${ }^{3}$, Deepak K S \\ ${ }^{1}$ Assistant Professor, Department of Anaesthesiology, M S Ramaiah Medical College and Hospital, \\ Bangalore - 560054, Karnataka, India, ${ }^{2}$ Professor, Department of Anaesthesiology, M S Ramaiah \\ Medical College and Hospital, Bangalore - 560054, Karnataka, India, ${ }^{3}$ Assistant Professor, \\ Department of Anaesthesiology M S Ramaiah Medical College and Hospital, Bangalore - 560054, \\ Karnataka, India, ${ }^{4}$ Resident, Department of Anaesthesiology, M S Ramaiah Medical College and \\ Hospital, Bangalore - 560054, Karnataka, India
}

\begin{abstract}
Background
Mallampati (MP) score is used to predict the ease of endotracheal intubation. Pregnancy is often associated with failed intubation during anaesthesia for caesarean delivery and contributes majorly to maternal complications that are related to anaesthesia. This study aimed to determine the change in MP grade over the course of pregnancy (first trimester, during labour and post-delivery)
\end{abstract}

\begin{abstract}
Methods
The study was conducted on 389 pregnant women in the first trimester. Demographic data, airway characteristics and MP score of the patients were recorded at different stages of pregnancy. Change in MP grade was assessed for those patients, who have delivered vaginally.
\end{abstract}

\begin{abstract}
Results
The mean age of the study subjects was $25.77 \pm 3.64$ years. There was a significant association of MP score with weight gain $(P<0.0001)$ and change in neck circumference $(P<0.0001)$. There was a significant change in the number of patients with MP grade 3 and 4 was noted from first trimester $(\mathrm{n}=14 ; 2.26 \%)$ to 48 hours post-delivery $(\mathrm{n}=78 ; 24.6 \%)$ $(P<0.01)$, showing a strong association between change in MP score and pregnancy.
\end{abstract}

\section{Conclusion}

There was a significant increase in the MP score over the course of pregnancy that may be attributed to gestational weight gain along with variations in the neck circumference in pregnant women. Hence, assessment of MP score is important for proper management of difficult intubation, especially in parturient.

Keywords: intubation, labour, pregnancy, weight gain

\section{Introduction}

The management of parturients poses complex challenges to anesthesiologists, including risk factors like pulmonary aspiration, emergency caesarean-section and balancing the conflicting

*Correspondence: Archana Shivashankar

E mail: archinddoc@yahoo.com

iD https://orcid.org/0000-0002-6165-2980

Received: 03/08/2020

Accepted: $13 / 04 / 2021$

DOI: http:/doi.org/10.4038/slja.v29i1.8653 needs of both the mother and fetus. For obstetric patients, endotracheal intubation is known to be the standard care for airway management. ${ }^{1}$ Pregnant women pose a high risk of failed intubation, constituting a challenge for the anesthesiologist. ${ }^{2}$ Mallampati (MP) grading has proven to be a primordial predictor of difficult tracheal intubation in parturients. ${ }^{3}$ The MP score gives a rough estimate of the size of tongue relative to the oral cavity. ${ }^{3}$

The incidence of failed intubation in obstetrics is higher when compared to general surgery and has not been well explained so far. ${ }^{4}$ Increase in MP score in parturients gives an insight into 
understanding the mechanisms involved in difficult intubation, majorly in parturients and immediately post labor. ${ }^{5}$

The MP classification allows for instantaneous identification of an increase in the tongue volume without the help of sophisticated devices. ${ }^{6}$ MP score may also change over the course of hours during the labor process and needs to be assessed prior to instrumentation. ${ }^{5}$ There are only few Indian studies on MP score in pregnant women. The score is not static, and it is important to evaluate the score frequently in obstetric patients to reduce the morbidity and mortality due to endotracheal intubation. ${ }^{1}$ This study aimed to determine the change in MP grade over the course of pregnancy (first trimester, during labor and post-delivery).

\section{Methods}

Five hundred pregnant women in the first trimester, aged $>18$ years, were enrolled and this study was conducted over a period of three years. Informed consent was procured from the patients and the study was approved by the institutional ethical committee (MSRMC/EC/2016), with CTRI registration number CTRI/2018/02/011944. Patients with multiple pregnancy, previous head and neck surgeries and temporomandibular disease were excluded. Women with initial MP grade 4 were excluded from enrollment because there is no description of a grade greater than 4 in MP classification. Demographics, mode of delivery, gravidity, and co-morbid disease of all the patients were noted. Figure 1 shows the consort flow diagram of recruitment of study participants.

The modified MP grade was assessed in all patients by the same anesthesiologist. ${ }^{7}$ The MP score was measured in a seated position, with head in neutral position, mouth opened widely with absence of phonation at five different time points, i.e. at first trimester, at the commencement/ induction of labor, during labor, after $24 \mathrm{~h}$ and $48 \mathrm{~h}$ of delivery. MP grade during labor was assessed after induction of labor, in the first stage of labor before giving labor analgesia, but before full cervical dilatation. Change in MP grade over different time points (first trimester to $48 \mathrm{~h}$ post-delivery) was assessed for those patients who delivered vaginally. Variables such as age, height, gravidity, mode of delivery, weight gain, change in thyromental distance (TMD) and neck circumference from first trimester to third trimester, volume of intravenous fluids infused during labor, and comorbid conditions associated with pregnancy (hypertensive disorders of pregnancy, hypothyroidism and gestational diabetes) were collected from patient's medical files or by in-person interviews.

Based on previous study, the change in MP grade during pregnancy, labor and post-delivery was reported to be $50 \% .{ }^{5}$ Hence, with a relative precision of $5.5 \%$ and desired CI of $95 \%$, sample size of 318 was required.

$\mathrm{R}$ v 3.6.0 was used to analyze the data. Continuous variables were represented by mean \pm SD while categorical variables were represented by frequency. Quade test was used to assess the change in MP score at two time points. Paired comparison was done with Wilcoxon signed rank test with BenjaminiHochberg adjustment whereas paired categorical variables using McNemar's test. $P \leq 0.05$ considered as statistically significant.

\section{Results}

Of 500 pregnant patients, only 389 were studied, as remaining were lost to follow-up. The mean age of participants was $25.77 \pm 3.64$ years and mean height was $155.3 \pm 4.0 \mathrm{cms}$. Characteristics of the participants are presented in Table 1.

Around $318(81.75 \%)$ patients underwent vaginal delivery. The distribution of MP grade during different stages of pregnancy and labor is shown in Figure 2. There was a significant relation between weight, neck circumference and TMD $(P<0.0001$; Table 2$)$ with MP score.

The MP score post-delivery was recorded for 318 patients who had vaginal delivery and assessed for change in MP score at different time points. In the first trimester, the number of patients $(n=304)$ with MP score of 1 and 2 (complete visualization of the soft palate, complete visualization of uvula) were greater than the number of patients with MP 1 and 2 during labor $(\mathrm{n}=235), 24 \mathrm{~h}$ post-delivery $(\mathrm{n}=232)$ and $48 \mathrm{~h}$ post-delivery $(\mathrm{n}=240)$, signifying a change in MP grade from first trimester to $48 \mathrm{~h}$ post-delivery, $P<0.0001$ (Table 3 ). A significant change in MP grade was noted 


\begin{tabular}{|c|c|c|c|}
\hline \multirow{2}{*}{\multicolumn{2}{|c|}{ parameters }} & \multicolumn{2}{|c|}{ Number of Participants $(n=389)$} \\
\hline & & Count (n) & Percent $(\%)$ \\
\hline \multirow{4}{*}{ Age } & $<20$ & 5 & 1.29 \\
\hline & $20-25$ & 203 & 52.19 \\
\hline & $26-30$ & 141 & 36.25 \\
\hline & $\geq 30$ & 40 & 10.28 \\
\hline \multirow{5}{*}{ Gravidity } & Primi & 201 & 51.67 \\
\hline & G2 & 139 & 35.73 \\
\hline & G3 & 41 & 10.54 \\
\hline & G4 & 7 & 1.8 \\
\hline & G5 & 1 & 0.26 \\
\hline \multirow{7}{*}{ Co-morbid disease } & Nil & 254 & 65.3 \\
\hline & Pregnancy induced hypertension & 48 & 12.34 \\
\hline & Gestational diabetes mellitus & 8 & 2.06 \\
\hline & Hypothyroid & 67 & 17.22 \\
\hline & $\begin{array}{l}\text { Pregnancy induced hypertension } \\
\text { and Hypothyroid }\end{array}$ & 1 & 0.26 \\
\hline & Tuberculosis & 1 & 0.26 \\
\hline & Anemia & 10 & 2.57 \\
\hline \multirow{2}{*}{ Mode of delivery } & Vaginal & 318 & 81.75 \\
\hline & Lower segment caesarean section & 71 & 18.25 \\
\hline Height $(\mathrm{cm})$ & \multicolumn{3}{|l|}{$155.3 \pm 4.0$} \\
\hline Intravenous Fluid & \multicolumn{3}{|l|}{$1021.54 \pm 408.24$} \\
\hline
\end{tabular}

Table 2

Correlation of different parameters with Mallampati score

\begin{tabular}{|l|c|c|}
\hline \multicolumn{1}{|c|}{ Parameters } & OR [95\% CI] & P-value \\
\hline Weight $(\mathrm{kg})$ & $1.03[1.02,1.04]$ & $<0.0001 * *$ \\
\hline $\begin{array}{l}\text { Neck } \\
\text { Circumference }(\mathrm{cm})\end{array}$ & $1.55[1.43,1.68]$ & $<0.0001 * *$ \\
\hline $\begin{array}{l}\text { Thyromental } \\
\text { Distance }(\mathrm{cm})\end{array}$ & $0.66[0.58,0.74]$ & $<0.0001 * *$ \\
\hline \multicolumn{3}{|l|}{} \\
**indicates statistically significant \\
\hline
\end{tabular}

at different time points from first trimester to pre labor $(P<0.0001)$. The number of pregnant women who had MP grade 3 in the first trimester was only $14(2.24 \%)$, which increased to $83(26.1 \%)$ during the pre-labor period.

The distribution of MP score at pre-labor, during labor, $24 \mathrm{~h}$ and post $48 \mathrm{~h}$ post-delivery was significantly different from first trimester $(P<0.0001)$. Distribution of MP score at $48 \mathrm{~h}$ post-delivery was significantly different from MP score at pre-labor $(P=0.0120)$, during labor $(P=0.0004)$ and $24 \mathrm{~h}$ post-delivery $(\mathrm{P}=0.0060)$. Participants with MP grade 3 and 4 were 85 $(26.8 \%)$ and $78(24.6 \%)$ at $24 \mathrm{~h}$ and $48 \mathrm{~h}$. post delivery, respectively, compared to only 14 (2.24\%) participants in the first trimester, which was statistically significant $(\underline{\mathrm{P}}<0.01)$.

\section{Discussion}

The MP score is derived from a simple airwayclassification system and is used to recognize the risk of difficult tracheal intubation. ${ }^{7}$ The MP classification includes four grades (1, 2, 3 and 4) depending on the soft palate visibility, ${ }^{8}$ with grade 4 being the worst.

During pregnancy there are multiple changes observed in the airway due to various factors, not all of them clearly defined. ${ }^{9}$ The physiological changes coherent with pregnancy and labor remarkably affect the respiratory system by decreasing lung volume and thoracic compliance and narrowing the upper airway. Anatomically, the upper airway is enveloped by soft tissues such as tongue and soft palate, which are encompassed by bony structures like the mandible and spine. Size of the airway space is determined by the balance between the size of the bony enclosure and the soft tissue volume. ${ }^{6}$ There is no obvious bone or joint deformity during pregnancy and labor. However, the soft tissue volume is increased, presumably due to pharyngeal edema and weight gain that occurs 
during pregnancy, ${ }^{5}$ which accounts for increasing grade of MP score during pregnancy and labor.

Similar to other study, we observed a significant increase in MP score from first trimester to prelabor and delivery ${ }^{5}$ in which there was a significant increase in MP score in patients from pre-labor to post labor (17 vs. $30 ; P<0.001)$, who had reached an MP score of 3-4.

In a previous study, ${ }^{10}$ the authors emphasized an association between airway change and weight gain during pregnancy. In the present study, using logistic regression, it was concluded that change in weight and change in neck circumference were significant factors affecting MP score change. For one unit increase in weight gain (per $\mathrm{kg}$ ), the odds of change in MP score increase by 1.04 times. In contrast, the study conducted by Boutonnet et al. ${ }^{11}$ did not find increased body weight to be predictive for airway changes, and this absence of correlation between weight gain and change in MP score may be explained by the fact that their first evaluation was done at 32 weeks of gestation.

Variation in neck circumference was also found to influence the MP grade. With one-unit increase $($ per $\mathrm{cm})$ in neck circumference, the odds of having an MP score of 3-4 was 1.55 times higher, akin to Lebouanlger et al. ${ }^{12}$ Lebouanlger et al. ${ }^{12}$ in their study using acoustic reflection method, have shown a significant diminution in the cross-sectional area of pharynx and subsequent increase in MP grade during normal pregnancy, with no change in the crosssectional area of the trachea. Our data were in agreement with these findings, which suggest that increase in neck circumference and weight gain during pregnancy lead to decrease in cross sectional area of the pharynx due to pharyngeal edema, increase in local fatty tissue volume and other features like generalized weight gain suggesting potential intubation. ${ }^{5}$ From the first trimester to other stages of pregnancy (pre delivery, during labor and post labor), the incidence of reduction in the number of patients with MP score 1-2 was $9.3 \%$.

This study has certain limitations. We did not use any form of airway imaging technique like reflectometry or photography to assess the MP classification. It was a subjective assessment performed by a single observer and the MP score of the patient in the previous stage of pregnancy were not blinded to the observer. Since it was a subjective measurement, the reliability of the assessment of MP score is questionable. Authors attempted to preclude this by instructing the participant thoroughly regarding maximum mouth opening and tongue protrusion without phonation before assessment.

\section{Conclusions}

Overall there is a significant increase in the MP score over the course of pregnancy. The changes in MP score can be seen due to various factors like weight gain, variations in the neck circumference and TMD. As several indications

Table 3

Mallampati score at different stages of pregnancy

\begin{tabular}{|c|c|c|c|c|c|}
\hline \multirow[b]{2}{*}{$\begin{array}{l}\text { Mallampati } \\
\text { score }\end{array}$} & \multicolumn{5}{|c|}{ Number of Patients $(n=318)$} \\
\hline & 1st Trimester & Pre-labor & During labor & $\begin{array}{c}24 \text { h Post- } \\
\text { Delivery }\end{array}$ & $\begin{array}{l}48 \text { h Post- } \\
\text { Delivery }\end{array}$ \\
\hline Grade 1 & 161 & 104 & 103 & 103 & 107 \\
\hline Grade 2 & 143 & 131 & 129 & 130 & 133 \\
\hline Grade 3 & 14 & 65 & 68 & 67 & 62 \\
\hline Grade 4 & - & 18 & 18 & 18 & 16 \\
\hline P-values & & $<0.0001^{\dagger, * * *}$ & $\begin{array}{c}<0.0001^{\dagger, * * *} \\
0.0898^{*}\end{array}$ & $\begin{array}{l}<0.0001^{\dagger}, * * * \\
0.1655^{\dagger} \\
1^{\S}\end{array}$ & $\begin{array}{c}<0.0001^{\dagger,,^{* * *}} \\
0.0120^{\ddagger *},{ }^{*} \\
0.0004^{\S, * *} \\
0.0060^{\dagger \dagger, * *}\end{array}$ \\
\hline \multicolumn{6}{|c|}{$\begin{array}{l}* * * \text { and } * * * \text { significant; }+ \text { indicates comparison of } 1 \text { st trimester with pre-labor, during labor, } 24 \mathrm{~h} \\
\text { post-delivery and } 48 \mathrm{~h} \text { post-delivery; } \neq \text { indicates comparison of pre-labor with during labor, } 24 \mathrm{~h} \text { post- } \\
\text { delivery and } 48 \mathrm{~h} \text { post-delivery; } \S \text { indicates comparison of during labor with } 24 \mathrm{~h} \text { and } 48 \mathrm{~h} \text { post- } \\
\text { delivery and }+\dagger \text { indicates comparison between } 24 \mathrm{~h} \text { post-delivery and } 48 \mathrm{~h} \text { post-delivery. }\end{array}$} \\
\hline
\end{tabular}


for general anesthesia still prevail in the obstetric population, the incidence of failed intubation remains high. Hence, it is important to evaluate the MP score especially prior to induction of anesthesia to avoid failed intubation. Assessment of MP score aids the anesthesiologist in identifying plausible risk of difficult intubation. This information is important to the anesthetic team during set up for a case and will influence the choice of equipment prepared for airway management.

\section{References}

1. Mushambi MC, Kinsella SM. Obstetric Anaesthetists' Association/difficult airway society difficult and

failed tracheal intubation guidelines--the way forward for the obstetric airway. British Journal of Anaesthesia 2015; 115(6): 815-8. https://doi.org/ 10.1093/bja/aev296 PMID: 26511060

2. Aydas AD, Basaranoglu G, Ozdemir H, Dooply SL, Muhammedoglu N, Kucuk S, et al. Airway changes in pregnant women before and after delivery. Irish Journal of Medical Science 2015; 184(2): 431

https://doi.org/ 10.1007/s11845-014-1138-8 PMID: 24859287

3. Roth D, Pace NL, Lee A, Hovhannisyan K, Warenits AM, Arrich J et al. Airway physical examination tests for detection of difficult airway management in apparently normal adult patients. Cochrane Database Systemic Reviews 2018; 5(5): CD008874. https://doi.org/10.1002/14651858.CD008874.pu b2

PMID: 29761867

4. King TA, Adams AP. Failed trachcal intubation. British Journal of Anaesthesia 1990; 65:400-414.

5. Kodali B-S, Chandrasekhar S, Bulich LN, Topulos GP, Datta S. Airway changes during labor and delivery. Anesthesiology 2008; 108(3): 357-62.

https://doi.org/10.1097/ALN.0b013e31816452d3 PMID: 18292672
6. Shiroh Isono. Mallampati Classification, an Estimate of Upper Airway Anatomical Balance, Can Change Rapidly during Labor. Anesthesiology 2008; 108(3): 347-349.

https://doi.org/10.1097/ALN.0b013e318164cb0b PMID: 18292667

7. Samsoon GL, Young JR. Difficult tracheal intubation: a retrospective study. Anaesthesia 1987; 42(5): 487-90.

https://doi.org/10.1111/j.1365-

2044.1987.tb04039.x

PMID 3592174

8. Aydogmus MT, Erkalp K, Sinikoglu SN, Usta TA, Ulger GO, Alagol A. Is ultrasonic investigation of transverse tracheal air shadow diameter reasonable for evaluation of difficult airway in pregnant women: A prospective comparative study. Pakistan Journal of Medical Sciences 2014; 30(1): 91-95.

https://doi.org/10.12669/pjms.301.3972 PMID: 24639838

9. Farcon EL, Kim MH, Marx GF: Changing Mallampati score during labor. Canadian Journal of Anaesthesia 1994; 41(1): 50-51.

10. Pilkington S, Carli F, Dakin MJ, Romney M, De Witt KA, DOR C et al. Increase in Mallampati score during pregnancy. British Journal of Anaesthesia. 1995; 74(6): 638-642. https://doi.org/10.1093/bja/74.6.638 PMID: 7640115

11. Boutonnet M, Faitot V, Katz A, Salomon L, Keita H. Mallampati class changes during pregnancy, labour, and after delivery: can these be predicted? British Journal of Anaesthesia 2010; 104(1): 67-70.

https://doi.org/10.1093/bja/aep356 PMID: 20007793

12. Leboulanger N, Louvet N, Rigouzzo A, M de Mesmay, Louis B, Farrugia M et al. Pregnancy is associated with a decrease in pharyngeal but not tracheal or laryngeal cross-sectional area: a pilot study using the acoustic reflection method. International Journal of Obstetric Anesthesia 2014; 23(1): 35-39.

https://doi.org/ 10.1016/j.ijoa.2013.08.008 PMID: 24333051 